\title{
JESS FIXATOR FOR HAND FRACTURES: OUR EXPERIENCE IN 20 PATIENTS
}

Rajendraprasad Butala ${ }^{1}$, Abhinav Garg 2 , Shikhar Singh 3, Praisy Garg 4 , Abhay Agarwal5, Nabokrishna Gohain 6 , Sunirmal Kumar Mukherjee ${ }^{7}$

${ }^{1}$ Associate Professor, Department of Orthopaedics, Dr. D. Y. Patil Medical College, Navi Mumbai.

${ }^{2}$ Registrar, Department of Orthopaedics, Dr. D. Y. Patil Medical College, Navi Mumbai.

${ }_{3}^{3}$ Senior Registrar, Department of Orthopaedics, Dr. D. Y. Patil Medical College, Navi Mumbai.

${ }_{4}^{4}$ Registrar, Department of Orthopaedics, Dr. D. Y. Patil Medical College, Navi Mumbai.

${ }_{5}^{5}$ Registrar, Department of Orthopaedics, Dr. D. Y. Patil Medical College, Navi Mumbai.

${ }^{6}$ Registrar, Department of Orthopaedics, Dr. D. Y. Patil Medical College, Navi Mumbai.

${ }^{7}$ Assistant Professor, Department of Orthopaedics, Dr. D. Y. Patil Medical College, Navi Mumbai.

\section{ABSTRACT}

\section{BACKGROUND}

The human hand has evolved into an organ of exceptional prehensile function, capable of highly complex movements and manipulation. Hand injury is extremely common and accounts for about $15 \%$ of the attendance at accidents and emergency departments.

\section{METHODS}

Various modes of treatment have been used for hand fractures, which include $\mathrm{K}$ wire fixation, mini plates, external fixator application.[1,2,3] We have retrospectively studied 20 patients with hand fracture (28 fractures) treated with Joshi's external stabilization system.[4] The aim of the study was principally to evaluate the functional outcome and duration of fracture healing. Functional outcome was assessed based on total active range of movement in degrees of each injured finger separately according to Duncan et al. Results: Overall, 12 phalangeal fractures had excellent result, 8 had good, 1 fair and 2 fractures had poor outcome. Among 4 metacarpal and 2 trapezium fractures treated, 1 had good outcome while the rest had excellent outcome. The results in our study were comparable with various other similar studies. Conclusion: From the results we can safely conclude that JESS is an effective alternative treatment for fractures of the hand providing excellent-to-good functional outcome.

\section{KEYWORDS}

JESS, Hand Fracture, Joshi External Fixator.

HOW TO CITE THIS ARTICLE: Butala R, Garg A, Singh S, et al. JESS fixator for hand fractures: our experience in 20 patients. J. Evolution Med. Dent. Sci. 2016;5(34):1946-1949, DOI: 10.14260/jemds/2016/459

\section{INTRODUCTION}

The human hand has evolved into an organ of exceptional prehensile function capable of highly complex movements and manipulation. Hands have got a very distinct and important role. It is an organ both for powerful grasp as in lifting heavy objects as well as delicate pinch and hook functions. Hand injury is extremely common and accounts for about $15 \%$ of the attendance at accidents and emergency departments. Fractures of metacarpals and phalanges are probably the most common fractures in the skeletal system. Some of the common causes of hand injuries are crush/compression injuries, blunt trauma, fall, road traffic accidents, machinery injury, sports related activity and explosions/fire arm injuries. Various modes of treatment have been used for hand fractures which include $\mathrm{K}$ wire fixation, mini plates, external fixator application.[1,2,3] In disabilities of the Hand, the finest surgery and after care is more essential than in any other region of the body. We have retrospectively studied 20 patients with hand fracture treated with Joshi's external stabilization system.[4]

Financial or Other, Competing Interest: None.

Submission 09-03-2016, Peer Review 05-04-2016,

Acceptance 11-04-2016, Published 28-04-2016.

Corresponding Author:

Dr. Sunirmal Kumar Mukherjee,

\#702, Torino, South Avenue,

Hiranandani Gardens,

Powai, Mumbai-400076.

Maharashtra, India.

E-mail: sunmukherjee1987@yahoo.com

DOI: $10.14260 /$ jemds $/ 2016 / 459$
The aim of the study was principally to evaluate the functional outcome and duration of fracture healing.

\section{AIMS AND OBJECTIVES}

To study the anatomical and functional outcome of fractures of the hands managed by Joshi's External Stabilization System.

\section{MATERIALS AND METHODS}

20 patients ( 17 men and 3 women) comprising 28 fractures of hand satisfying the inclusion criteria were treated by Joshi's external stabilizing system in our institute between January 2013 and December 2015. The patients were followed at regular interval and the results were evaluated clinically and radiologically.

\section{Inclusion Criteria}

- $\quad$ Skeletally mature patients.

- $\quad$ Closed and Open fractures (Type 1, 2 and 3A).

- Intra-articular fractures.

\section{Exclusion Criteria}

- Pathological fractures.

- $\quad$ Crush hand with multiple compound Grade 3 fracture.

- Isolated fracture of distal phalanx.

- $\quad$ Associated co-morbidities.

Pre-operative Assessment

- History.

- Routine investigations. 
- $\quad$ Pre-operative X-rays - AP, lateral/oblique view.

- Pre-anaesthetic check-up and clearance.

- In case of open fractures, debridement of the wound and thorough irrigation was done with normal saline.

\section{Surgery}

- Closed reduction was achieved by traction and manipulation.

- To maintain reduction, external fixator was applied by passing at least two $\mathrm{K}$ wires proximally and two $\mathrm{K}$ wires distal to the fracture.

- These two constructs were connected using connecting rods.

- Distraction was given if required.

- Image intensifier was used as a guide for the steps mentioned above.

\section{Post-Operative}

- The patients were taught active mobilization of the unaffected fingers, elbow and shoulder from immediate post-op period.

- $\quad$ Pin tract dressings were done regularly.

- $\quad$ Patients were called for periodic evaluation at 2 weeks, 4 weeks, 6 weeks and 8 weeks on OPD basis to assess:

- Stability of fixation.

- Tenderness at fracture site.

- Pin tract infections.

- Residual stiffness.

JESS removal was done at 4 to 6 weeks interval with immediate vigorous mobilization of the immobilized joint to avoid stiffness.

Functional outcome was assessed based on total active range of movement in degrees of each injured finger separately according to Duncan et al.

\begin{tabular}{|c|c|c|}
\hline Finger & Thumb & Result \\
\hline 220 to 260 & 119 to 140 & Excellent \\
\hline 180 to 219 & 98 to 118 & Good \\
\hline 130 to 179 & 70 to 97 & Fair \\
\hline$<130$ & $<70$ & Poor \\
\hline \multicolumn{3}{|c|}{ Table 1 } \\
\hline
\end{tabular}

\section{OBSERVATION AND RESULTS}

Of 20 patients, 14 had trauma resulting from road traffic accident (11 had fall from bike and 2 suffered car accident, 1 pedestrian hit by bicycle) and 6 were from household trauma or occupation related trauma. Two patients had carpal bone fracture - trapezium fracture. Associated injuries seen were contralateral clavicle fracture middle third (1 patient), proximal humerus fracture (Undisplaced - 1 patient managed conservatively), tibial midshaft fracture (1 patient).

\section{The Mean Follow-Up was up to 6 Months}

The device had been removed at a mean of 4.6 weeks after a phalangeal fracture ( 4 to 6 weeks) and 5.2 weeks after a metacarpal fracture ( 4 to 8 weeks); 8 fractures showed complications during the period of fixation.

In 4 patients, pins had become loose. Pin loosening was seen at the earliest by 4 weeks (Mean duration of pin loosening -4.5 weeks). Loosening of a pin was managed by removal of the device because the fractures had healed. In one case, loosening resulted in displacement of the fracture, which required surgical revision and was fixed using percutaneous $\mathrm{K}$-wire and immobilization for another 6 weeks.

One patient developed stiffness of hand - reflex sympathetic dystrophy. Passive assisted mobilization was started immediately followed by gradual active mobilization. The patient regained pre-trauma functional activity by 8 weeks.

In 1 patient the fracture became displaced, which required revision. In 2 patients, there was infection of the wounds which was treated by local debridement and antibiotics. Both the cases eventually healed completely.

The mean period of treatment for phalangeal fractures was 4 months and for metacarpal fractures 3 months, by which time patients regained full functional activity of hand.

- All fractures healed without further operation.

- After removal of the pin, there were no cases with sinus formation or other signs of infection.

\begin{tabular}{|c|c|c|c|}
\hline Result & Phalangeal & Metacarpal & $\begin{array}{l}\text { Carpal Bones } \\
\text { (Trapezium) }\end{array}$ \\
\hline Excellent & 12 & 03 & 02 \\
\hline Good & 08 & 01 & 00 \\
\hline Fair & 01 & 00 & 00 \\
\hline Poor & 01 & 00 & 00 \\
\hline \multicolumn{4}{|c|}{ Table 2 } \\
\hline
\end{tabular}

Results of treatment in 22 phalangeal fractures, 4 metacarpal fractures and 2 carpal fractures. (Result monitored at 6 month follow-up)

\section{PATIENT 1}

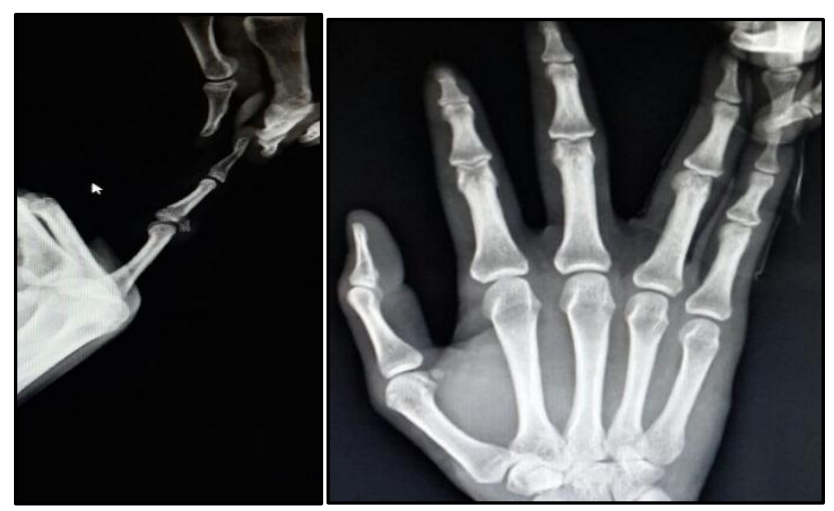

Fig. 1: Pre-op X-Ray of Patient 1

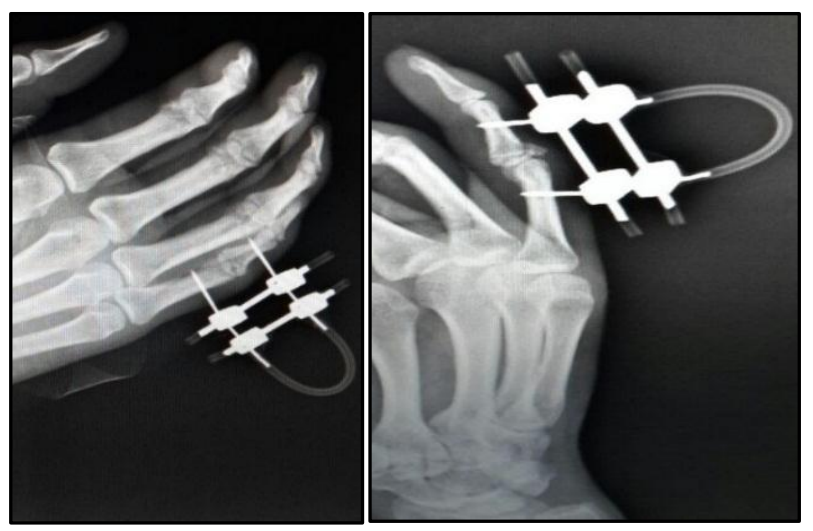

Fig. 2: Post-op X-Rays 


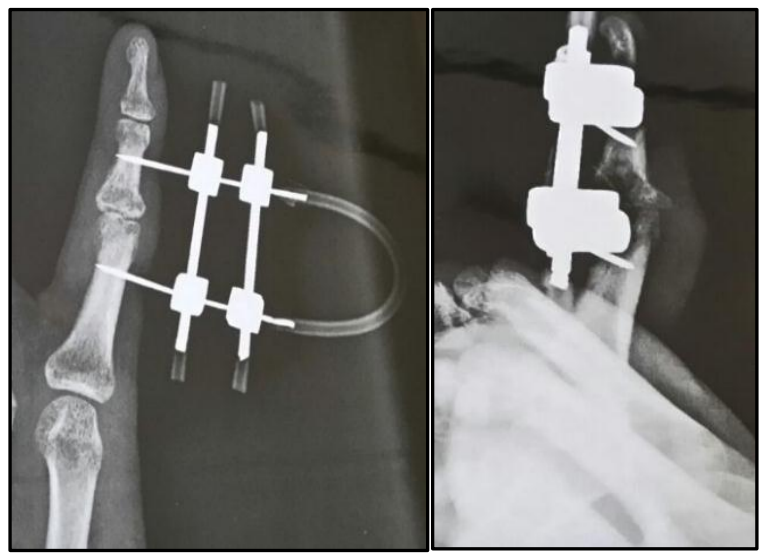

Fig. 3: Five Weeks Post-Op X-Rays

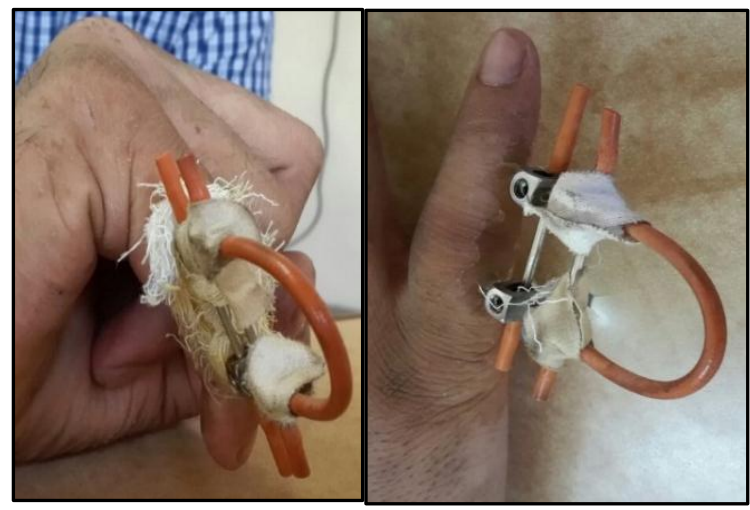

Fig. 4: Clinical Pics of the Same Patient

\section{PATIENT 2}

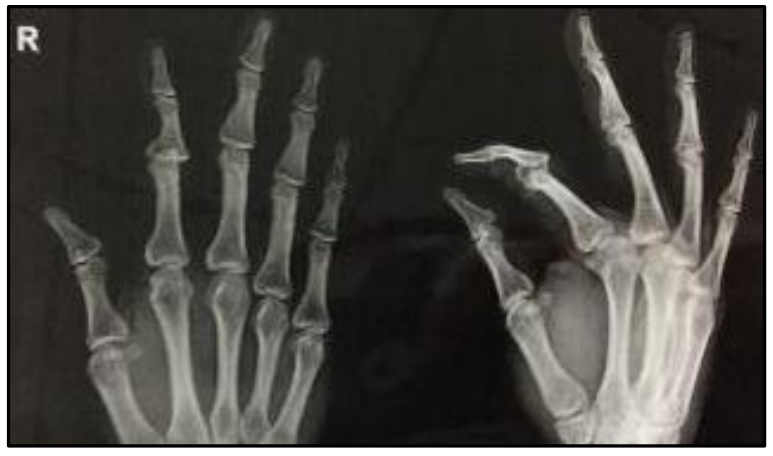

Fig. 5: Pre-Op X-Ray

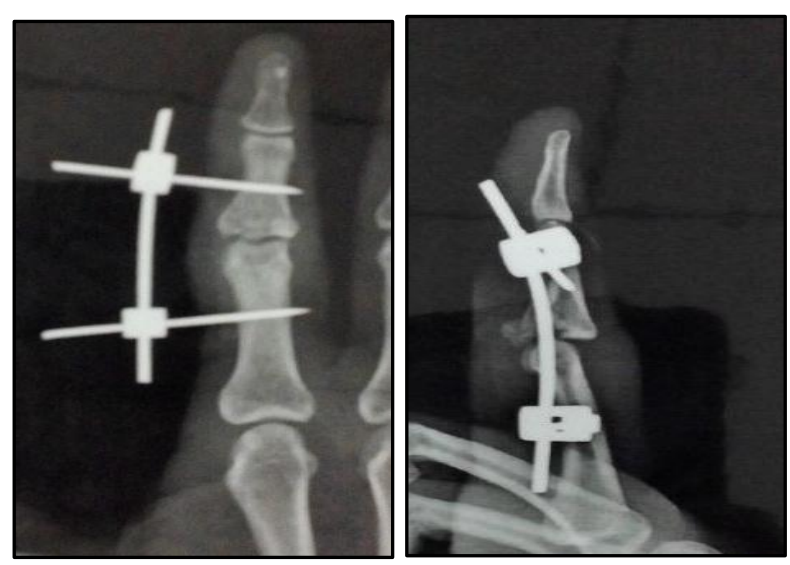

Fig. 6: Post-op X-Ray



Fig. 7: Post-Op 4 Weeks X-Ray

PATIENT 3

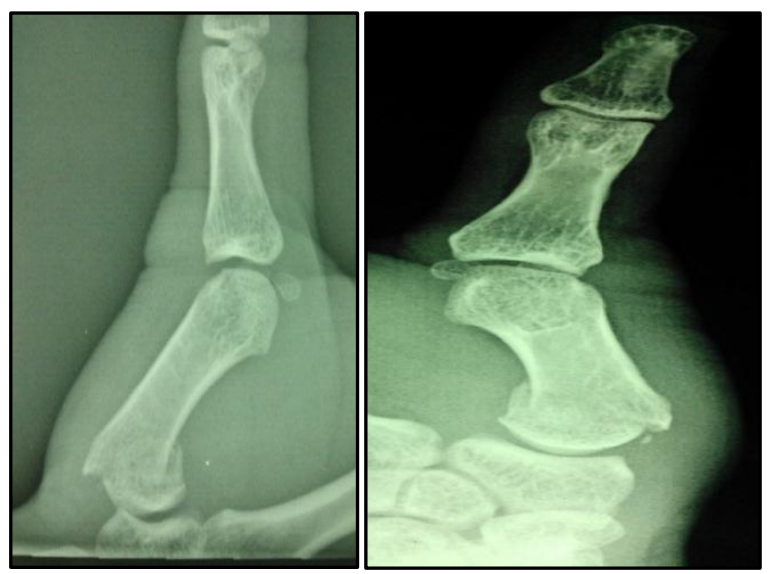

Fig. 8: Pre-Op X-Ray

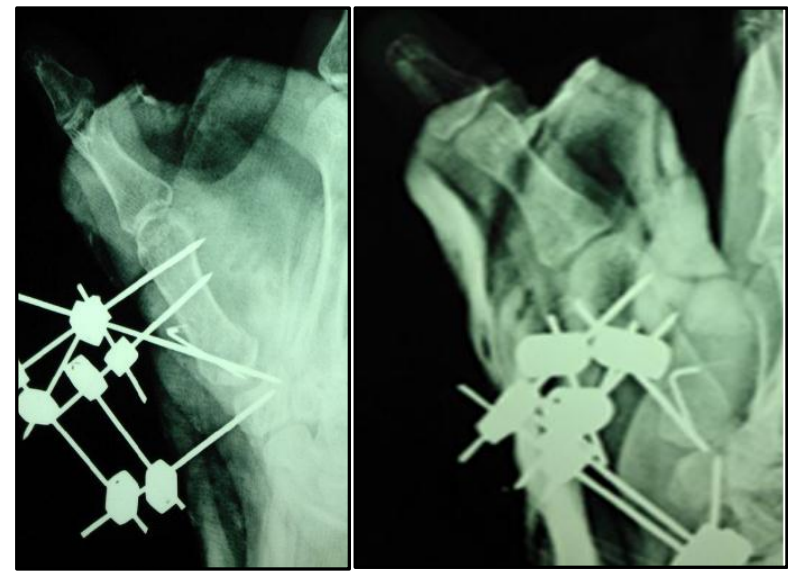

Fig. 9: Post-Op X-Ray

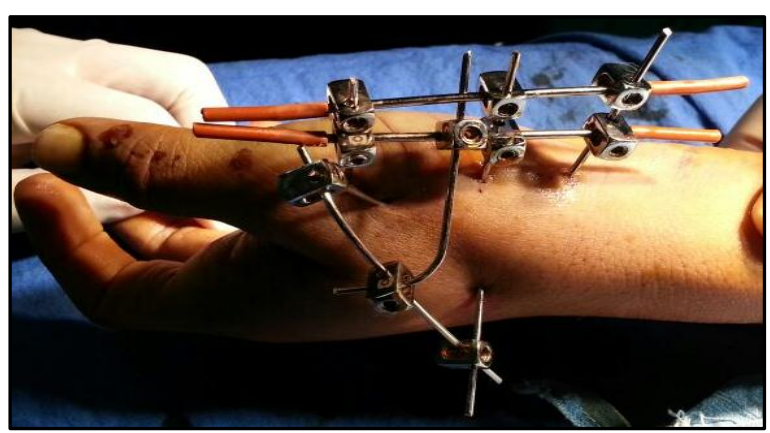

Fig. 10: A Multiplanar JESS Frame for Base of $1^{\text {st }}$ Metacarpal Fracture 


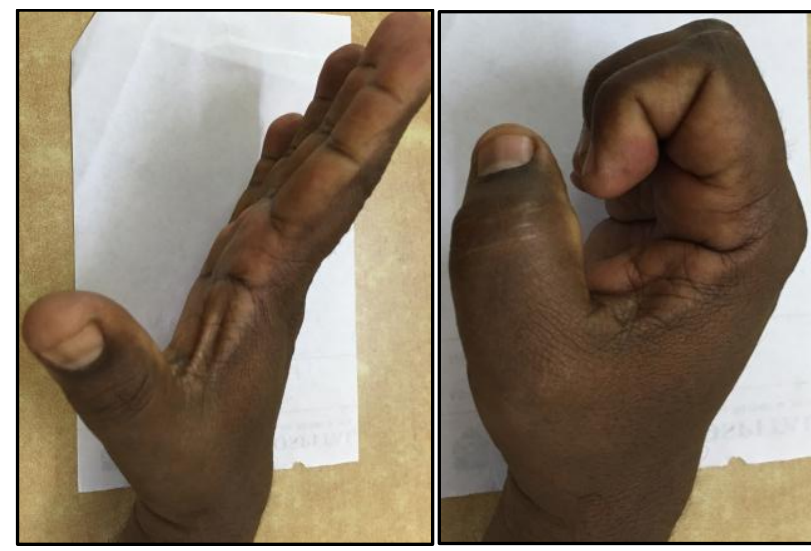

Fig. 11: Full Range of Motion as Compared to Adjacent Finger at 6 Months (Operated for Ring Finger JESS)

\section{DISCUSSION}

Fractures of metacarpals and phalanges are probably the most common fractures in the skeletal system and are often neglected as minor injuries. Most of the fractures are treated conservatively, but some form of fixation is often indicated in unstable fractures, intra-articular fractures, open fractures and multiple fractures.

Different types of JESS frame were applied in our study. JESS frame acts by the principle of ligamentotaxis to achieve closed reduction without requiring immobilization of adjacent joint. In our institute, 20 cases (17 men and 3 women) of fractures of hand were treated by JESS. Most injuries were caused by road traffic accidents followed by fall of heavy object on hand and machinery related injuries; 22 phalangeal fractures and 4 metacarpal fractures and 2 trapezium fractures were seen.

Functional outcome was assessed based on total active range of movement in degrees of each injured finger separately according to Duncan et al. All patients were followed up for 6 months post-operative period. There were no general complications. Phalangeal JESS frame ( $4.6 \mathrm{wks}$.) were removed early as compared to metacarpal JESS (5.2 wks.). The JESS frame applied for trapezium fracture were removed at 6 weeks post-operative period. In 4 patients, pins had become loose.

Only two complications were seen, one had stiffness of hand due to reflex sympathetic dystrophy and other had pin loosening due to pin tract infection with loss of reduction both causing poor outcome at 8 weeks follow-up. However, the patient with hand stiffness regained full functional outcome by 4 months. The other patient with pin loosening subsequently developed stiffness at the proximal and distal interphalangeal joint despite vigorous attempts of joint mobilisation resulting in poor functional outcome even at 6 months post-op. Overall,
12 phalangeal fractures had excellent result, 8 had good, 1 fair and 2 fractures had poor outcome. Among 4 metacarpal fractures treated, 3 had excellent outcome and 1 had good outcome. Both the trapezium fracture treated had excellent functional outcome.

The results in our study were comparable with various other studies. Parson et al[5] also did a prospective study of 30 patients, out of which 26 were male and 4 female, with the mean age of 28 yrs. They reported union in all their patients, with metacarpal fractures (Mean duration 4.8 weeks) and phalangeal fractures (Mean duration 4.5 weeks). Schuind F et al[6] conducted a study comprising of 26 patients (21 males and 5 females). In their study, most of their patients had bony union within 12 weeks. Mullet et al[7] removed device at a mean duration of 6 weeks and also observed union in all patients, but after a much longer duration of 28 weeks.

The study carried out by us had 20 patients and the results achieved by us were comparable to the studies mentioned above.

\section{CONCLUSION}

From the results, we can safely conclude that JESS is an effective alternative treatment for fractures of the hand. It is cheap and easily available. Technically, also it is less demanding. It also reduces surgical trauma and protects the vascular integrity and has good-to-excellent functional outcome.

\section{REFERENCES}

1. Crockett DJ. Rigid fixation of bones of the hand using $\mathrm{K}$ wires bonded with acrylic resin. Hand 1974;6(1):106-7.

2. Cziffer E. Static fixation of finger fractures. Hand clini 1993;9(4):639-50.

3. Fitoussi F, Ip WY, Chow SP. External fixation for comminuted phalangeal fractures a biomechanical cadaver study. J Hand surg Br 1996;21(6):760-764.

4. Joshi BB. Percutaneous internal fixation of fractures of the proximal phalanges. Hand 1976;8(1):86-92.

5. Parsons SW, Fitzgerald JA, Sherare JR. External fixation of unstable metacarpal and phalangeal fractures. J Hand Surg Br 1992;17(2):151-5.

6. Schuind F, Donkerwolcke M, Burny F, et al. External minifixation for treatment of closed fractures of the metacarpol bone. J Ortho Traumat 1991;5(2):146-52.

7. Mullet JH, Synott K, Noel J, et al. Use of the S quattro dynamic external fixation in eth treatment of difficult hand fractures. J Hand Surg Br 1999;24(30):350-4. 